\title{
Non-cancer female fertility preservation: strategy for a birth
}

\begin{abstract}
Since 2011, French bioethics law has allowedoocyte vitrification as a daily tool for medically assisted reproduction for infertility as well as for fertility preservation in women with a high risk of fertility loss. This fertility preservation is now part of the medical proposals offered during cancer treatment but not yet in benign pelvic diseases such as endometriosis. We can now report our first birth after oocyte vitrification for severe hyperalgic pelvic endometriosis. Preserving female gamete to prevent fertility loss represents a progress that should be offered by centers which are skilled in oocyte vitrification. As with on cofertility, fertility preservation in cancer-free patients has to be protocolized in multidisciplinary staff meeting.
\end{abstract}

Keywords: oocyte vitrification, fertility preservation, endometriosis
Volume 9 Issue 4 - 2018

\section{Pierre Boyer, Cendrine Siraudin, Marie Gervoise-Boyer,Vanessa Lubin, Aurélie Amar Hoffet}

Department of Medicine and Biology of Reproduction, SaintJoseph-de-Marseille Hospital, France

Correspondence: Pierre Boyer, Department of Medicine and Biology of Reproduction, Saint-Joseph-de-Marseille Hospital, 26, boulevard de Louvain, I 3008 Marseille, France,

Email: pboyer@hopital-saint-joseph.fr

Received: March II, 2018 | Published: July 27, 2018

\section{Summary}

In recent years, the law $n^{\circ} 2011-814$ of July 7, 2011 relating to the bioethics authorizes the ultra-rapid freezing of the oocytes offering to the centers of medical assistance with procreation a new tool at the service of the preservation of the female fertility. Although the medical proposal to resort to preservation has taken its place in cancerous pathologies, the same is not the case for other situations of risk of impaired fertility, whether medical or surgical. Endometriosis, which we know the impact on fertility, is a perfect example of the need to set up a treatment course with cryo - oocytes conservation for young patients before surgical management. We report the first birth after treatment for preservation of fertility in the context of severe hyperalgic endometriosis. The meeting between the MPA and the pelvic gynecological surgery is a step forward for these women. We will discuss the implementation of a protocol of care in the image of what exists on fertility.

\section{Introduction}

The Bioethics Laws of $2004^{1}$ stipulate that "any person whose medical care is likely to impair fertility or whose fertility may be prematurely impaired can benefit from the collection and conservation of gametes or Germinal tissue, for the further realization of Assisted Reproductive Technologies (ART) for their benefit, or in hopes of preserving and restoring their fertility.

The French Centers for the Study and Conservation of the Egg and Sperm (CECOS) have for a very long time proposed an effective method of spermatozoa freezing. However, for women, freezing and storage of oocytes is much more recent due to the necessity of vitrification technical control. After validating our technique as part of the field of Assisted Reproductive Technologies (ART), we proposed this method for fertility preservation. Today, this method which is based on a proven methodology, validated in Evidence Based Medicine, ${ }^{2}$ fulfills the ethical imperatives of such a medical proposal, as early as 2013. ${ }^{3}$ We take care of patients in an Oncological context but also of those who have benign pathologies. Among these situations, endometriosis is a disease with a high risk of premature ovarian failure and iterative surgery. ${ }^{4,5}$ In our center endometriosis represents the majority of cancer-free patient care. The parental project of one of our patients has become a current issue and thus allowing us to announce the first birth in fertility preservation for severe hyperalgic pelvic endometriosis. Beyond this particular case, this approach led us to analyze the management of fertility preservation for non-cancer pathologies, which do not yet benefit from any specific protocol.

\section{Clinical case}

Ms E ..., 27 years old and single, was supported for iterative surgery for severe hyperalgic endometriosis with deep peritoneal and ovarian lesions. In 2010, she underwent a cystectomy and ablation of nodules of Douglas' cul-de-sac, then, in 2014, surgery was performed once again, but this time for hyperalgia, leading to a recurrence of cystectomy and adhesiolysis. She was thereafter addressed to our center by her surgeon due to a high risks of recurrence and of ovarian reserve alteration. Clinical history report edmenarches occurring from the age of 12, with regular and short cycles of 25 days, associated with hyperalgic menstrual syndrome and deep dyspareunia. On another note, the patient was healthy with a body mass index of 18.21 and moderate smoking of one pack per week. The ovarian reserve was estimated by antral follicular count (AFC) at 14 and the antiMüllerian hormone (AMH) assay at 1.62ng/ml. Given the persistence of a hyperalgic syndrome and considering the inherent risk of iterative surgery, her case was studied for fertility preservation. The multidisciplinary staff meeting (MSM) gavea favorable outcome to this request, but since the patient wasn't available, due to personal reasons, the prescribed preservation cycle was not immediately carried out until 2015, when the patient, who was no more single, finally came back with her husband and a desire for children. Clinically, hyperalgesia syndrome was still present; the vaginal touch still indicated painful uterine mobilization and athickening of Douglas'culde-sac. At the ultrasound, the ovaries appeared modified with multiple endometriosic zones. Considering this symptomatology and the desire for pregnancy, the MSM suggested a fertility preservation management associating in vitro fertilization with Intra Cytoplasmic Sperm Injection (IVF-ICSI)and a partial vitrification of there sulting 
oocyte cohort using Kitazato vitrification and thawing solutions and cryotop device. Ovarian stimulation was carried out according to a short antagonist protocol with Follitropine alpha (Gonal F®) 225IU/ day for 11 days, Ganirelix (Orgalutran ${ }^{\circledR}$ ) was introduced on the 6th day of the stimulation (S6), triggering by Gonadotrophin 5000IU on the $11^{\text {th }}$ day and on the $13^{\text {th }}$ day collection of 18 oocytes in metaphase II was carried out by transvaginal puncture under ultrasound control. The risk of a hyper stimulation syndrome re-evaluated on the day of the puncture finally required a "freeze all" strategy: it was decided to transform the partial attempt into a total freezing of the 18 mature oocytes by giving up embryo implantation due to the risk of a decompensation for the patient. Rest and medical follow-up allowed the patient to recover quickly without hospitalization. The first Cycle of Oocyte Warming (COW) was programmed in March 2016during a spontaneous cycle, 6 oocytes were reheated, 6 were injected, and 5 oocytes were fertilized with the presence of 2 Pronuclear (PN) 18 hours later. A single blastocyst was obtained and replaced in utero without success on the $5^{\text {th }}$ day (D5). The programming of a second cycle of oocyte warming was decided in May 2016during a spontaneous cycle. Ultrasound monitoring and dosing determined ovulation with a 19$\mathrm{mm}$ follicle at $\mathrm{S} 10$ and a Lutein Hormone (LH) assay at $44 \mathrm{IU} / \mathrm{ml}$ at S11. The warming of 6 oocytes was programmed at S12, 5 oocytes were injected, and 4 oocytes were fertilized with the presence of 2 PN. Two ongoing embryos were replaced in utero at D5. Pregnancy was confirmed with a first $\beta \mathrm{hC}$ Gassay at $55 \mathrm{IU} / \mathrm{ml}$ with a second assay, 3 days later at $226 \mathrm{IU} / \mathrm{ml}$. The first ultrasound echography showed a single embryonic sac with cardiac activity. The pregnancy continued without any complications, leading to the birth, in January 2017 (at 37.5 weeks of amenorrhea), of a baby girlin good health, weighing $2840 \mathrm{~g}$ for $46.5 \mathrm{~cm}$.

\section{Discussion}

\section{Implementation of our activity}

Since our center has been authorized for fertility preservation, we have studied 54 demands not related to cancer. This activity was structured according to the same management regimen as onco fertility: any file had to be documented and discussed in MSM before inclusion. To date, 18 requests have been refused and 36 have been accepted: 28 patients have already received at least 1 oocyte puncture and 8 cases are ongoing. The 36 cancer-free patients who were selected were divided into: 25 cases of recurrent endometriosis, 7 cases of benign recurrent ovarian cysts, 3 cases of premature ovarian failure and 1 case of vasculitis before immunosuppressive treatment. We preserved 10.7 oocytes on average (4-30) in 28 patients who accepted the preservation proposal and performed at least one stimulation cycle. We offered up to 3 cycles of stimulation and some of these patients are still under care to increase their number of cryopreserved oocytes.

This case report illustrates female fertility preservation in a cancer-free patient which brings us to the opportunity to discuss a medical recommendation that did not exist just a few months ago. Oocyte freezing by vitrification has opened this new application field. Preservation of the mature oocyte provides the best conditions for survival and fertilization and thus the best potential for fertilized embryos.

\section{Characteristics of the treatment for a cancer-free population.}

The medical situation of a cancer-free population is incomparable to the population in oncology: the life expectancy and the waiting time before patient care are favorably different. Rational multidisciplinary organization has less of a time constraint but the proposal must still be rapid and appropriate. Our intervention presents a risk of ovarian hyper stimulation. In practice, the absence of an emergency context allowed us to consider the use of several cycles ( 1 to 3 ) to optimize the number of preserved oocytes. The minimum useful number of oocytes preserved for future pregnancy still needs to be clarified. Early data suggested that 8 to 10 oocytes for a woman under the age of 36 would allow for about a $50 \%$ chance of birth. ${ }^{6}$

\section{The specific case of endometriosis}

Endometriosis is as disease with a complex physio pathogenicity, which alters women's fertility by: peritoneal fluid inflammation, increase of the cytokines, pelvic adhesions and endometrial receptivity impairment. Endometriosis also alters the ovarian function by declining the reserve, with the production of free iron, and by progressive stretching of the cortex by the cyst regardless of the surgery. ${ }^{7}$ Its prevalence concerns $5-10 \%$ of women of childbearing age $^{8}$ and $50 \%$ of those women of childbearing age with endometriosis will require ART. ${ }^{9}$

Surgical treatment of Endometriosis is an integral part of patient care but can be deleterious to the ovarian patrimony especially if there is ovarian damage..$^{10}$ Iterative surgery does not improve fertility. ${ }^{11}$ and the risk of recurrence of the disease is high: $30 \%$ to $50 \%,{ }^{12}$ even in simple adhesiolysis cases. The consequences may range from Premature Ovarian Failure (POF), ${ }^{13}$ with decreased $\mathrm{AMH}$, to early menopause in immediate post-surgery. ${ }^{14} \mathrm{New}$ techniques seem promising: $\mathrm{CO}_{2}$ laser, sclera therapy or Plasma-energy. Patients should be informed of the risks according to their age, their ovarian reserve and the presence of cysts or not. Oocyte preservation in endometriosis has its rightful place. The frozen ovarian cortex transplant after bilateral ovariectomy on endometriosis..$^{15}$ has traditionally been proposed, but is not adapted to all patients. Oocyte vitrification could be proposed in situations of risk of $\mathrm{POF}^{5}$ and before age 36 , if possible. ${ }^{6}$

Indications such as: the presence of bilateral endometrioma, the history of cystectomy with contra lateral recurrence and the relevance of a second surgery could be discussed in MSM.

Nevertheless endometriosis represents a relatively common disease and including affected women is still a matter of debate due to logistics and financial effects. ${ }^{5}$

\section{A useful and necessary protocol}

In the context of fertility preservation for cancer-free patients, the assessment of the pathology, therapeutic proposals, infectious serological assessment, evaluation of the ovarian reserve with AFC and $\mathrm{AMH}$, clinico-biological consultations, seeking free and informed consent with a reflection period, and discussion in MSM validating the patient care and programming treatments, are all essential steps for a good patient health care path. An established protocol highlights concrete elements complementing surgical MSM organized around endometriosis. ${ }^{16}$ This approach model can be adapted for other chronic diseases affecting women of reproductive age. For example, recurrent mucinous or dermoid cysts are part of the indications. Seven of our patients were able to benefit from preservation after cystectomy or unilateral ovariectomy in this context. 


\section{Conclusion}

The scope of fertility preservation is expanding with the control of oocyte freezing. Chronic recurrent non-cancer pathologies can also be concerned by this progress. In this case ART teams are more and more sought out by medical and surgical teams to complete patient care. ${ }^{17,18}$ Endometriosis illustrates the value of seeing patients as quickly as possible before the first surgical procedure or at least before a second look. In light of our center's first experiment, we recommend using the ART team at the earliest stage of surgical management so the fertility proposal can be evaluated in MSM and then organized according to a specific care path.

\section{Acknowledgements}

None.

\section{Conflicts of interest}

The author declares that they do not have any conflicts of interest.

\section{References}

1. LOIS. Law No. 2004-800 of 6 August 2004 on bioethics. Official Journal of the French Republic. 2004:1-92.

2. Cobo A, Diaz C. Clinical application of oocyte vitrification: a systematic review and meta-analysis of randomized controlled trials. Fertil Steril. 2011;96(2):277-85.

3. Boyer P, Rodrigues P, Tourame P, et al. Third Millennium Assisted Reproductive Technologies: The Impact of Oocyte Vitrification. In: Shevach Friedler, editor. Europe: InTech; 2012. 156 p.

4. Decanter C, d'Argent EM, Boujenah J, et al. Endometriosis and fertility preservation: CNGOF-HAS Endometriosis Guidelines. Gyn Obst Fertil Senol. 2018;46(3):368-72.

5. Somigliana E, Vigano P, Filippi F, et al. Fertility preservation in women with endometriosis: for all, for some, for none?. Hum Reprod. 2015;30(6):1280-6.

6. Cobo A, García-Velasco JA, Coello A, et al. Oocyte vitrification as an efficient option for elective fertility preservation. Fertil Steril. 2016;105(3):755-64.
7. Sanchez AM, Vigano P, Somigliana E, et al. The distinguishing cellular and molecular features of the endometriotic ovarian cyst: from patho physiology to the potential endometrioma-mediated damage to the ovary. Hum Reprod Update. 2014;20(2):217-30.

8. Vigano P, Parazzini F, Somigliana E, et al. Endometriosis: epidemiology and etiological factors. Best Pract Res Clin Obstet Gynaecol. 2004;18(2):177-200.

9. De Ziegler D, Borghese B, Chapron C. Endometriosis and infertility: patho physiology and management. Lancet. 2010;376(9742):730-8.

10. Kitajima M, Defrere S, Dolmans MM, et al. Endometriomas as a possible cause of reduced ovarian reserve in women with endometriosis. Fertil Steril. 2011;96(3):685-91.

11. Vercellini P, Somigliana E, Vigano P, et al. The effect of second-line surgery on reproductive performance of women with recurrent endometriosis: a systematic review. Acta Obstet Gynecol Scand. 2009;88(10):1074-82.

12. Brosens I, Gordts S, Benagiano G. Endometriosis in adolescents is a hidden, Progressive and severe disease that deserves attention, not just compassion. Hum Reprod. 2013;28(8):2026-31.

13. Coccia ME, Rizzello F, Mariani G, et al. Ovarian surgery for bilateral endometriomas influences age at menopause. Hum Reprod. 2011;26(11):3000-7.

14. Busacca M, Riparini J, Somigliana E, et al. Postsurgical ovarian failure after laparoscopic excision of bilateral endometriomas. Am J Obstet Gynecol. 2006;195(2):421-5.

15. Donnez J, Squifflet J, Dolmans MM, et al. Orthotopic transplantation of fresh ovarian cortex: areport of two cases. Fertil Steril. 2005;84(4):1018.

16. Collinet P, Fritel X, Revel-Delhom C, et al. Guidelines Management of endometriosis CNGOF/HAS clinical practice guidelines - Short version. J Gynecol Obstet \& Human Reprod. 2018.

17. Ulrich U, Buchweitz O, Greb R, et al. National German Guideline: Guideline for the diagnosis and treatment of endometriosis. Geburtsh Frauenheilk. 2014;74(12):1104-18.

18. Elizur S, Chian R, Holzer H, et al. Cryopreservation of oocytes in a young woman with severe and symptomatic endometriosis: a new indication for fertility preservation. Fertil Steril. 2009;91(1):293e1-3. 